\title{
Efficient fiber to SOI photonic wire coupler fabricated using standard CMOS technology
}

\author{
G. Roelkens ${ }^{1}$, Student Member IEEE, P. Dumon, Student Member IEEE, W. Bogaerts, Student \\ member IEEE, D. Van Thourhout, Member IEEE, R. Baets, Senior Member IEEE \\ Ghent University-IMEC, Department of Information Technology- Sint-Pietersnieuwstraat 41,9000 Gent, \\ Belgium \\ ${ }^{1}$ e-mail: gunther.roelkens@intec.ugent.be
}

\begin{abstract}
We present a Silicon-on-Insulator spot size converter fabricated using $248 \mathrm{~nm}$ deep UV lithography. The loss of the taper structure is around $1 \mathrm{~dB}$ over more than $60 \mathrm{~nm}$ wavelength range while the overall coupling loss from a lensed fiber into a $590 \mathrm{~nm}$ wide SOI waveguide was measured to be $1.9 \mathrm{~dB}$.
\end{abstract}

\section{INTRODUCTION}

Silicon-on-Insulator (SOI) is a very promising platform to fabricate nanophotonic optical components on a wafer scale using standard CMOS processes [1]. Photonic wire waveguides in silicon-on-insulator confine light within submicron dimensions by total internal reflection. While efficient devices are being demonstrated, the coupling of light from a fiber in and out of the nanophotonic waveguide remains a problem due to the large mode mismatch between the fiber mode and the SOI waveguide mode. Different approaches are being used to overcome this problem. Grating couplers are good candidates due to the lack of the need for cleaved facets. However they intrinsically suffer from a compromise between efficiency and optical bandwidth, making them unsuitable in some applications [2]. Another approach presented in literature is to use a spot size converter to transform the SOI waveguide mode to a polymer waveguide mode which matches a lensed fiber mode as shown in Fig. 1. [3] Optical bandwidth is

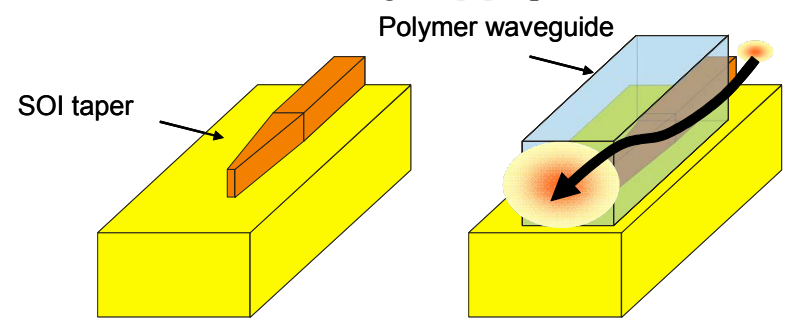

Fig. 1. SOI waveguide spot size converter for efficient coupling to a lensed fiber typically very large $(>100 \mathrm{~nm})$ and efficiencies are high $(<1 \mathrm{~dB}$ loss $)$. However, to obtain this high efficiency the width of the SOI taper tip needs to be below 100nm due to the strong optical confinement in the SOI waveguide. While this is not a problem for an e-beam lithography system in a research environment, this is much more difficult to achieve for the standard industrial CMOS deep UV lithography machines. Therefore, the design of the spot size converter needs to be adjusted to be able to fabricate them on an industrial scale.

\section{DESIGN OF DUV DEFINABLE SOI WAVEGUIDE TAPER}

The efficiency of the spot size converter was simulated using FIMMPROP 3D, a commercial fully vectorial eigenmode expansion tool. If we consider only the mode mismatch loss at the interface between the taper tip end and the polymer waveguide, we can calculate the upper limit of the achievable efficiency of the spot size converter. The polymer waveguide core was assumed to be $3 \mu \mathrm{mx} 3 \mu \mathrm{m}(\mathrm{n}=1.67)$ while the refractive index of the waveguide upper cladding is 1.54. The

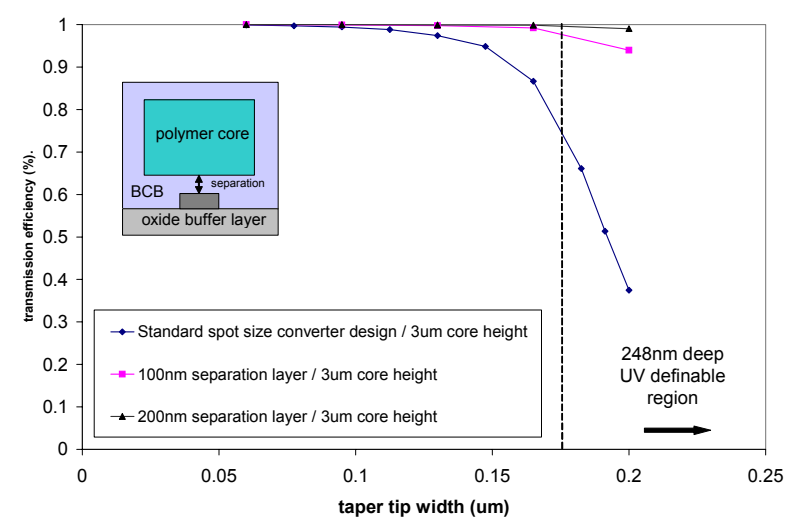

Fig. 2. Maximum spot size converter efficiency as a function of taper tip width 


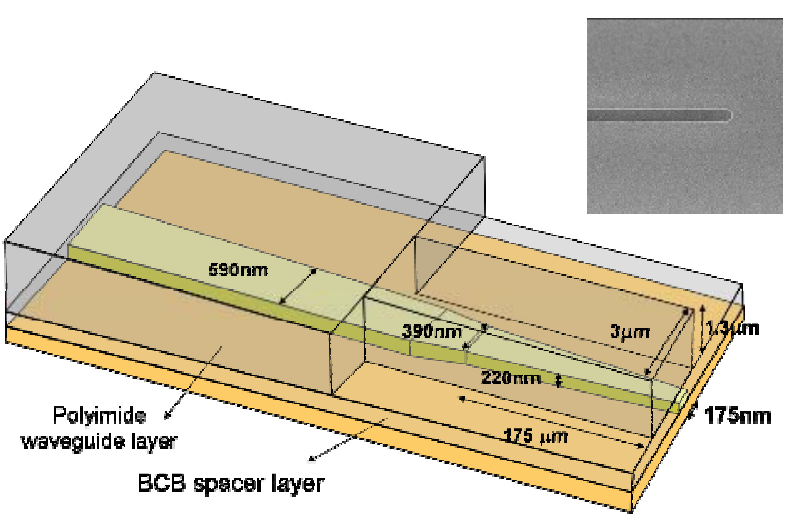

Fig. 3. Design of an SOI spot size converter and SEM picture of a fabricated taper tip

results are shown in Fig. 2. Because the minimal achievable taper tip size using a $248 \mathrm{~nm}$ deep UV lithography system is around $175 \mathrm{~nm}$, there is a substantial efficiency reduction compared to the sub $100 \mathrm{~nm}$ taper tips achievable using e-beam machines. To overcome this problem, an additional spacer layer is added to reduce the coupling between the waveguide modes as shown in the inset of Fig. 2. The influence of the spacer layer thickness on coupling efficiency of the spot size converter is also shown. It can be seen that the efficiency of the taper can be enhanced by adding this spacer layer.

\section{FABRICATION AND MEASUREMENTS}

Piecewise linear Silicon-on-Insulator spot size converters were fabricated using an ASML PAS5500/750 stepper. The SOI waveguide dimensions are shown in Fig. 3, together with an SEM picture of the taper tip. After waveguide fabrication a $200 \mathrm{~nm}$ benzocyclobutene (BCB) film was spin coated on top of the SOI waveguide structure. After curing, a polyimide waveguide core layer of $1.3 \mu \mathrm{m}$ thick was applied and the waveguide core was etched through using a $100 \mathrm{~nm}$ Ti mask and ICP plasma etching. After removal of the Ti mask a thick BCB top cladding was applied. The refractive index of the polyimide and $\mathrm{BCB}$ for $\mathrm{TE}$ polarization at $1.55 \mu \mathrm{m}$ is 1.67 and 1.54 respectively, characterized using a Metricon 2010 prism coupling setup. The structure used to characterize the spot size converters is shown in Fig. 4. A grating coupler was used to inject light into the fundamental TE waveguide mode of the SOI waveguide, while a lensed fiber with a spotsize of $2.5 \mu \mathrm{mx} 2.5 \mu \mathrm{m}$ or an objective lens was used to collect the light at the polymer waveguide facet. The grating coupler used in the experiments was characterized to have $7 \mathrm{~dB}$ loss and a $60 \mathrm{~nm} 3 \mathrm{~dB}$ bandwidth. Fig. 5 shows a transmission spectrum

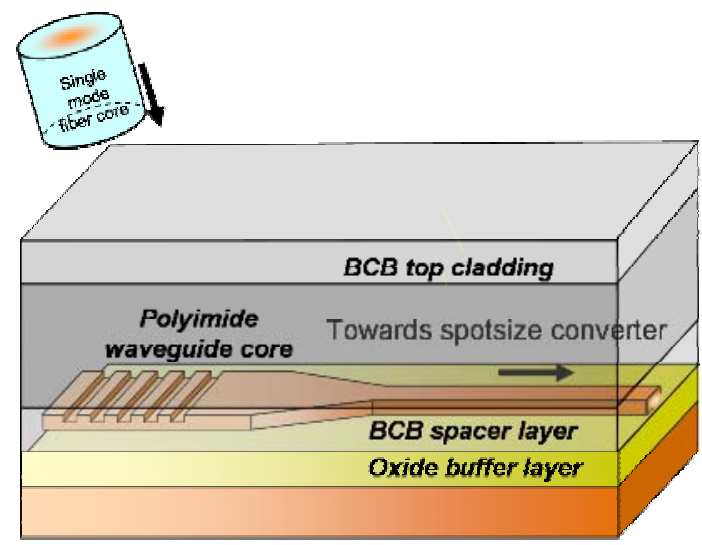

Fig. 4. Spot size converter measurement setup - fundamental mode excitation by grating coupler

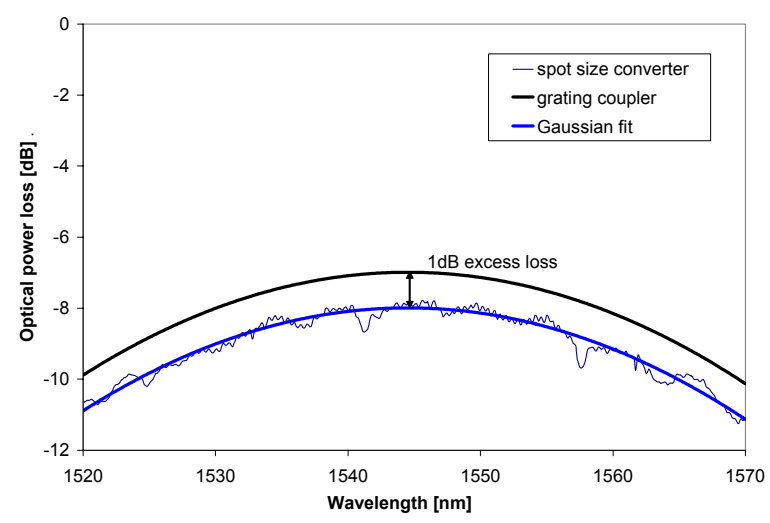

Fig. 5. Measured transmission spectrum with a superimposed grating transmission spectrum

measured by light collection using an objective lens. The coupling efficiency of the grating coupler is superimposed. This implies that the SOI spot size converter itself shows $1 \mathrm{~dB}$ loss. The coupling loss from a lensed fiber into a $590 \mathrm{~nm}$ wide SOI waveguide was measured to be $1.9 \mathrm{~dB}$. The $0.9 \mathrm{~dB}$ extra loss is caused by the mode mismatch between the polymer waveguide mode and the lensed fiber spot and the additional specified $0.5 \mathrm{~dB}$ loss of the lensed fiber used.

\section{REFERENCES}

[1] W. Bogaerts, R. Baets, P. Dumon, V. Wiaux, S. Beckx, D. Taillaert, B. Luyssaert, J. Van Campenhout, P. Bienstman and D. Van Thourhout, "Nanophotonic waveguides in silicon-on-insulator fabricated with CMOS technology", Journ. of Lightwave Technology, Vol. 23(1), pp. $401-412$, Jan 2005

[2] D. Taillaert, W. Bogaerts, P. Bienstman, T.F. Krauss, P. Van Daele, I. Moerman, S. Verstuyft, K. De Mesel and R. Baets, “An outof-plane grating coupler for efficient butt-coupling between compact planar waveguides and single-mode fibers", Journal of Quantum Electronics, Vol. 38 (7), pp. 949 - 955, July 2002

[3] T. Shoji, T. Tsuchizawa, T. Watanabe, K. Yamada and H. Morita, "Spot-size converter for low-loss coupling between 0.3um square $\mathrm{Si}$ wire waveguides and single mode fibers", $15^{\text {th }}$ annual meeting of the IEEE Lasers and Electro-Optics Society, vol. 1, p.289-290, 2002 